\title{
ADDITIVE COMMUTATORS BETWEEN $2 \times 2$ INTEGRAL MATRIX REPRESENTATIONS OF ORDERS IN IDENTICAL OR DIFFERENT QUADRATIC NUMBER FIELDS
}

BY OLGA TAUSSKY ${ }^{1}$

Communicated March 18, 1974

The following theorem holds:

THEOREM 1. Let $A, B$ be two integral $2 \times 2$ matrices. Let the characteristic roots of $A$ be $\alpha, \alpha^{\prime}$ and let the characteristic roots of $B$ be $\beta, \beta^{\prime}$, all assumed irrational. Then the determinant of

$$
L=A B-B A
$$

is a negative norm in both $Q(\alpha), Q(\beta)$.

REMARK. The proof of this theorem gives an algorithmic procedure for expressing an integer as a norm in a quadratic field.

Proof. There exists ${ }^{2}$ an integral matrix $S$ with the property that $S^{-1} A S$ is the companion matrix

$$
\left(\begin{array}{cc}
0 & 1 \\
-\operatorname{det} A & \operatorname{tr} A
\end{array}\right)
$$

of $A$. Since the companion matrix has the characteristic vectors $(1, \alpha)^{\prime}$, $\left(1, \alpha^{\prime}\right)^{\prime}$ the matrix $T=\left(\begin{array}{ll}1 & 1 \\ \alpha & \alpha^{\prime}\end{array}\right)$ has the property that $T^{-1} S^{-1} A S T=\left(\begin{array}{c}{ }^{\alpha} \\ \alpha^{\prime}\end{array}\right)$. Apply then the same similarity also to $B$ and to $L$, i.e. to (*). Let the outcome of this be denoted by

$$
\left(\begin{array}{cc}
\alpha & \\
& \alpha^{\prime}
\end{array}\right) B^{(\alpha)}-B^{(\alpha)}\left(\begin{array}{cc}
\alpha & \\
& \alpha^{\prime}
\end{array}\right)=L^{(\alpha)}=\left(\begin{array}{ll}
0 & l_{2} \\
l_{3} & 0
\end{array}\right)
$$

then $l_{2}, l_{3}$ are elements in $Q(\alpha)$.

Apply the similarity defined by $T^{-1}$ to $L^{(\alpha)}$. The result must be rational. A straightforward computation using the fact that $\alpha, \alpha^{\prime}=-\frac{1}{2}(\operatorname{tr} A \pm \sqrt{ } m)$, with $m=\left(\operatorname{tr} A^{2}-4 \operatorname{det} A\right)$, shows that

$$
\left(\begin{array}{cc}
1 & 1 \\
\alpha & \alpha^{\prime}
\end{array}\right)\left(\begin{array}{cc}
0 & l_{2} \\
l_{3} & 0
\end{array}\right)\left(\begin{array}{cc}
\alpha^{\prime} & -1 \\
-\alpha & 1
\end{array}\right) \frac{1}{\alpha^{\prime}-\alpha}=-\frac{1}{\sqrt{ } m}\left(\begin{array}{cc}
\alpha^{\prime} l_{3}-\alpha l_{2} & l_{2}-l_{3} \\
\alpha^{\prime 2} l_{3}-\alpha^{2} l_{2} & -\alpha^{\prime} l_{3}+\alpha l_{2}
\end{array}\right) .
$$

AMS (MOS) subject classifications (1970). Primary 15A36, 12A50, $10 \mathrm{C} 10$.

${ }^{1}$ This work was carried out in part under an NSF contract.

${ }^{2}$ For further information in the number theoretic case on this see [1]. 
This implies

$$
\begin{aligned}
& l_{2}-l_{3}=r_{1} \sqrt{ } m, \text { with } r_{1} \text { rational, } \\
& -m^{-1 / 2}\left[\frac{1}{2}(\operatorname{tr} A-\sqrt{ } m) l_{3}-\frac{1}{2}(\operatorname{tr} A+\sqrt{ } m) l_{2}\right] \\
& =-m^{-1 / 2}\left[\frac{1}{2} \operatorname{tr} A\left(l_{3}-l_{2}\right)-\frac{1}{2} \sqrt{ } m\left(l_{3}+l_{2}\right)\right] \\
& =\text { rational. }
\end{aligned}
$$

In virtue of (1) we obtain

$$
l_{2}+l_{3}=r_{2}, \text { with } r_{2} \text { rational. }
$$

From (1), (3) follows

$$
l_{2}=\frac{1}{2}\left(r_{2}+r_{1} \sqrt{ } m\right), \quad l_{3}=\frac{1}{2}\left(r_{2}-r_{1} \sqrt{ } m\right) .
$$

Hence $l_{2}, l_{3}$ are conjugate elements in $Q(\alpha)$. Since

$$
\operatorname{det}\left(\begin{array}{ll}
0 & l_{2} \\
l_{3} & 0
\end{array}\right)=-l_{2} l_{3}=\operatorname{det} L,
$$

the theorem follows if it is further observed that $A B-B A=-(B A-A B)$ and that $\operatorname{det}(A B-B A)=\operatorname{det}(B A-A B)$.

THEOREM 2. Let $Z$ be a matrix of the form $\left(\begin{array}{ll}0 & 1 \\ m & 0\end{array}\right)$ when $m$ is an integer not a square. If $Z$ is expressed in the form $X Y-Y X$, where $X, Y$ are rational matrices, ${ }^{3}$ then the characteristic roots of $X$ lie in the field $Q(\sqrt{ } M)$ where $M$ is the norm of an element in $Q(\sqrt{ } m)$.

It can further be shown that $M$ can be chosen as an arbitrary norm in $Q(\sqrt{ } m)$. Combining this fact with Theorem 1 leads to the following result:

THEOREM 3. Every negative norm in a quadratic field can be represented as $\operatorname{det}(A B-B A)$.

EXAMPLES.

1. $A=\left(\begin{array}{ll}0 & 1 \\ m & 0\end{array}\right), B=\left(\begin{array}{ll}0 & 1 \\ n & 0\end{array}\right), A B-B A=\left(\begin{array}{cc}n-m & 0 \\ 0 & m-n\end{array}\right)$.

2. $A=\left(\begin{array}{ll}0 & 1 \\ m & 0\end{array}\right), B=\left(\begin{array}{ll}1 & 1 \\ 0 & 1\end{array}\right)\left(\begin{array}{ll}0 & 1 \\ n & 0\end{array}\right)\left(\begin{array}{rr}1 & -1 \\ 0 & 1\end{array}\right)$,

$$
\begin{aligned}
A B-B A & =\left(\begin{array}{cc}
n-m+m n & -2 n \\
2 m n & -n+m-m n
\end{array}\right), \\
\operatorname{det}(A B-B A) & =-\left[(n-m+m n)^{2}-4 m n^{2}\right] \\
& =-\left[(m-n+m n)^{2}-4 m^{2} n\right] .
\end{aligned}
$$

\footnotetext{
${ }^{3}$ This is always possible by a theorem of Shoda.
} 
3. A random choice.

$$
A=\left(\begin{array}{ll}
1 & 2 \\
3 & 4
\end{array}\right), \quad B=\left(\begin{array}{ll}
1 & 3 \\
1 & 5
\end{array}\right)
$$

$A$ has characteristic polynomial $x^{2}-5 x-2$ and roots in $Q(\sqrt{ }(33))$. $B$ has characteristic polynomial $x^{2}-6 x+2$ and roots in $Q(\sqrt{ } 7)$. The commutator $A B-B A$ has determinant

$$
-58=-\operatorname{norm}(31+\sqrt{ }(33)) / 4=-\operatorname{norm}(11+3 \sqrt{ } 7) .
$$

REMARK. Zassenhaus observed that for matrices $A$ with $A^{2}=\operatorname{det} A \cdot I$ the relation $A L+L A=0$ holds. This can be generalized to the fact that the operator defined via $A$ on the space of $2 \times 2$ matrices has the characteristic vector $L$ with respect to the characteristic root trace $A$.

\section{REFERENCE}

1. O. Taussky, A result concerning classes of matrices, J. Number Theory 6 (1974), $64-71$.

Department of Mathematics, California Institute of Technology, Pasadena, CALIFORNIA 91109 